\title{
Search for low mass BSM particles using $h(125)$ at CMS
}

\section{Chayanit Asawatangtrakuldee ${ }^{* \dagger}$}

Deutsches Elektronen-Synchrotron (DESY), Hamburg

E-mail: chayanitecern.ch

\begin{abstract}
Searches for low mass beyond the Standard Model (BSM) particles using the discovered Higgs boson at $125 \mathrm{GeV}$ are presented in different possible channels, including invisible decays of the Higgs boson, the Higgs boson decays to new light bosons in which the light bosons decay to SM particles, and Lepton Flavour Violation (LFV) of the Higgs boson. The data is collected with the CMS detector at the LHC which corresponding to integrated luminosities of 19.7 and $2.3 \mathrm{fb}^{-1}$ at central-of-mass energies of 8 and $13 \mathrm{TeV}$, respectively. No excess of signal is observed. Upper limits are placed on the branching fractions of the Higgs boson decay to different BSM particles, assuming the SM cross sections. As well, the branching fractions of LFV Higgs boson are given. In addition, the combination of potential invisible decay modes of the Higgs boson is done for all channels and the results are interpreted in the context of Higgs-portal dark matter models.
\end{abstract}

XXV International Workshop on Deep-Inelastic Scattering and Related Subjects

3-7 April 2017

University of Birmingham, $U K$

\footnotetext{
* Speaker.

${ }^{\dagger}$ on behalf of the CMS Collaboration
} 


\section{Introduction}

The discovery of the Higgs boson at $125 \mathrm{GeV}$, henceforth referred to as $\mathrm{h}(125)$, and the studies of its properties by the ATLAS and CMS Collaborations [1,2,3] at the CERN LHC have placed major constraints on potential models of new physics beyond the Standard Model (BSM). Although all measurements to date indicate compatibility with the SM Higgs boson, the SM alone cannot address several crucial issues, such as the hierarchy problem or the nature of dark matter. Many theories beyond the Standard Model (BSM), therefore, have been proposed to address all these questions. In addition, the present uncertainties from the couplings measurements are large, and the possibility for non-SM properties of the Higgs boson remains. This would be a strong sign of new physics beyond the SM.

The branching fraction of $\mathrm{h} \rightarrow Z Z \rightarrow 4 v$ in the SM is very small but invisible Higgs boson decays are possible in a wide range of BSM models, for example through decays to neutralinos in supersymmetric models, or graviscalars in models with extra dimensions. Precision measurements of the couplings of the Higgs boson from a combination of the 7 and $8 \mathrm{TeV}$ ATLAS and CMS datasets provide indirect constraints on additional contributions to the Higgs boson decay width from non-SM decay processes. The resulting indirect upper limit on the Higgs boson branching fraction to non-SM decays is $34 \%$ at the $95 \%$ confidence level (CL) [4]. Direct searches for invisible decays of the Higgs boson are possible by requiring that the Higgs boson recoils against a visible system. The typical signatures at the LHC include the most sensitive channel, vector boson fusion production (VBF), associated production $(\mathrm{VH})$, and gluon fusion production $(\mathrm{ggH})$.

Two-Higgs-doublet models (2HDM) are an extension of the SM by introducing two Higgs doublets which, after symmetry breaking, lead to five physical Higgs states. The lightest scalar of $2 \mathrm{HDM}$ can be compatible with the SM-like properties of the discovered Higgs boson in the decoupling limit, where all other scalars have larger masses. A complex $S U(2)_{L}$ singlet field $S$ can be added to $2 \mathrm{HDM}$, with a small mixing with the doublets; such a model is called $2 \mathrm{HDM}+\mathrm{S}$. This leads to two additional singlet states, a CP-odd scalar $a$ and a CP-even $s$, which inherit a mixture of the Higgs doublets fermion interactions. In such a model, also known as NMSSM, the branching fraction of the Higgs boson to a pair of $a$ or $s$ bosons can be sizeable, and a wide variety of exotic Higgs decays is allowed [5], especially $h \rightarrow a a$.

In the SM, lepton flavour violating (LFV) decays of the Higgs boson are forbidden if the theory is to be renormalizable. If this requirement is relaxed, such that the theory is valid only to a finite mass scale, then LFV couplings may be introduced. LFV decays can occur naturally in models with more than one Higgs doublet without abandoning renormalizability. They also arise in supersymmetric models, composite Higgs boson models, Randall-Sundrum models, and many others. The presence of LFV couplings would allow $\mu \rightarrow e, \tau \rightarrow \mu$, and $\tau \rightarrow e$ transitions to proceed via the exchange of a virtual Higgs boson $[6,7]$.

In this document, we review searches for low mass BSM particles using $\mathrm{h}(125)$ performed by the CMS experiment with 7, 8 and $13 \mathrm{TeV}$ data. 


\section{Search for invisible decays of the $h(125)$}

\subsection{VBF channel}

This production mode is characterised by the presence of two jets with a large separation in $\eta$ and a large invariant mass $\left(m_{j j}\right)$. The contributions from the dominant $\mathrm{Z}(v v)+\mathrm{jets}$ and $\mathrm{W}(l v)+\mathrm{jets}$ backgrounds and the QCD multijet backgrounds are estimated using control regions in data. A simultaneous fit to the yields in the signal and control regions is performed to extract any potential signal and place upper limits on $\mathrm{B}(\mathrm{h} \rightarrow \mathrm{inv})$. The observed (expected) upper limit on the invisible branching fraction of the $\mathrm{h}(125)$ boson, assuming the SM production cross section, is found to be $69 \%(62 \%)$ at $95 \%$ confidence level (CL) corresponding to integrated luminosity of $2.3 \mathrm{fb}^{-1}$ at $\sqrt{s}$ $=13 \mathrm{TeV}$, as presented on Figure.1 (left) [8].
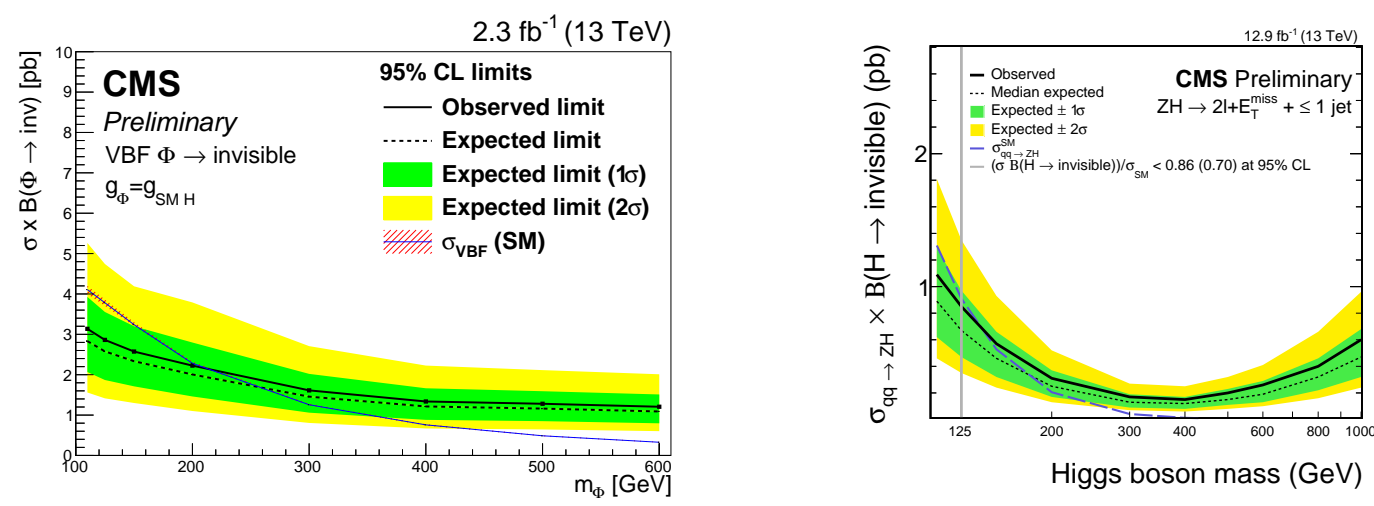

Figure 1: Left: Observed and expected 95\% CL limit on $\sigma \times \mathrm{B}(\Phi \rightarrow$ inv $)$ as a function of scalar mass $\left(m_{\Phi}\right)$ assuming the couplings of the scalar $\left(g_{\Phi}\right)$ to vector bosons to be the same as a Higgs boson with the same mass. Right: Observed and expected 95\% CL limit on the $\mathrm{ZH}$ production cross section times invisible branching fraction, $\sigma_{Z H} \times \mathrm{B}(\mathrm{H} \rightarrow$ inv $)$ as a function of the Higgs boson mass. SM couplings are assumed.

\section{2 $\mathrm{Z}(l l) \mathrm{H}$ channel}

The $\mathrm{ZH}$ production mode, where the $\mathrm{Z}$ boson decays to a pair of charged leptons, has a smaller cross section than VBF but a clean final state with lower background. The search targets events with a pair of same-flavour, opposite-charge leptons $(l=e, \mu)$, consistent with a leptonic $\mathrm{Z}$ boson decay, produced in association with a large missing transverse energy $\left(E_{T}^{\text {miss }}\right)$. The background is dominated by the diboson processes, $Z Z \rightarrow l l v v$ and $W Z \rightarrow l v l l$, which contribute roughly $70 \%$ and $25 \%$ of the total background, respectively. The observed (expected) upper limit on the invisible branching fraction of the $\mathrm{h}(125)$ boson, assuming the SM production cross section, is found to be $86 \%(70 \%)$ at $95 \% \mathrm{CL}$ corresponding to integrated luminosity of $12.9 \mathrm{fb}^{-1}$ at $\sqrt{s}=13 \mathrm{TeV}$, shown on Figure.1 (right) [9].

\section{$2.3 \mathrm{~V}(j j) \mathrm{H}$ and ggH channels}

The search strategies for the $\mathrm{V}(j j) \mathrm{H}$ mode, in which the vector boson decays hadronically, and ggH modes are very similar, targeting events with large $E_{T}^{\text {miss }}$, with the missing transverse 
momentum $\left(p_{T}^{m i s s}\right)$ recoiling against jets from either gluon radiation or a hadronically decaying vector boson. The dominant backgrounds arise from $\mathrm{Z}(v v)+$ jets and $\mathrm{W}(l v)+\mathrm{jets}$ events, accounting for $90 \%$ of the total background. These backgrounds are estimated using control regions in data and a simultaneous fit to the $E_{T}^{\text {miss }}$ distribution of the events across all regions is performed to extract a potential signal. Finally, the observed (expected) upper limit on the invisible branching fraction of the $\mathrm{h}(125)$ is set at $44 \%(56 \%)$ at $95 \%$ confidence level corresponding to integrated luminosity of $12.9 \mathrm{fb}^{-1}$ at $\sqrt{s}=13 \mathrm{TeV}[10]$.

\subsection{Combination of $\mathbf{h}(\mathbf{1 2 5}) \rightarrow$ invisible measurements}

The limits are obtained from the simultaneous analysis of all categories and from sub-combinations of categories, which target each of the ggH, VBF, and VH production mechanisms. Observed and expected upper limits on $\sigma \mathrm{B}(\mathrm{h} \rightarrow \mathrm{inv}) / \sigma(\mathrm{SM})$, where $\sigma(\mathrm{SM})$ is the total SM Higgs boson production cross section, are determined at the 95\% CL and presented in Fig. 2 (left). They provide comparable results with indirect upper limits of the couplings of the $\mathrm{h}(125)$ boson to non-SM decays. Moreover, the upper limit on $\mathrm{B}(\mathrm{h} \rightarrow$ inv $)$, under the assumption of SM production cross sections for the Higgs boson, can also be interpreted in the context of a Higgs-portal model of DM interactions [11], as shown in Fig.2 (right). These combined results are based on data corresponding to integrated luminosities of 5.1, 19.7, and $2.3 \mathrm{fb}^{-1}$ at centre-of-mass energies of 7, 8, and 13 $\mathrm{TeV}$, respectively [12].
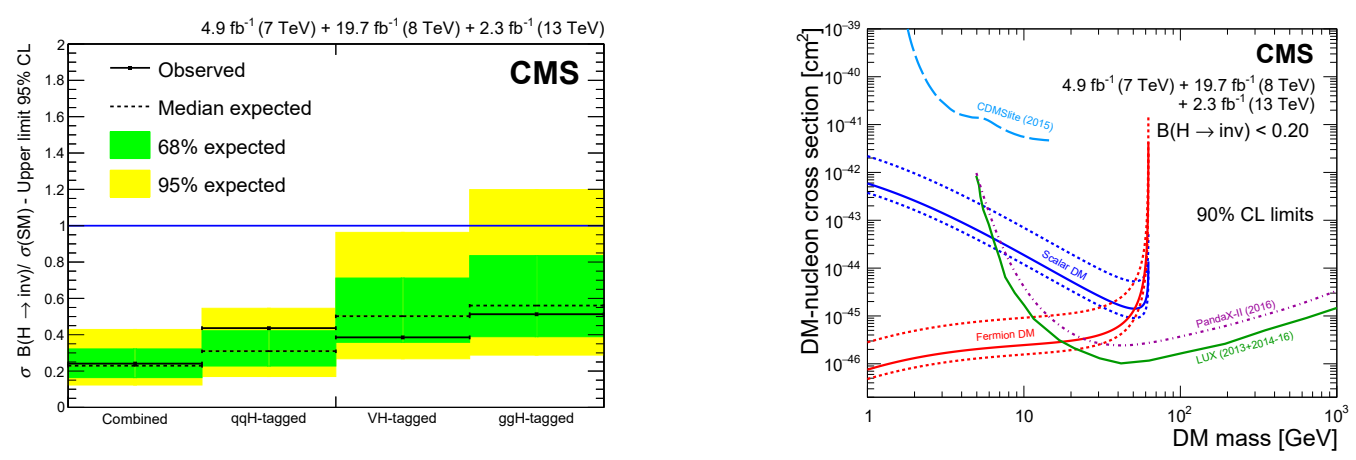

Figure 2: Left: Observed and expected upper limits on $\sigma \cdot \mathrm{B}(\mathrm{h} \rightarrow \mathrm{inv}) / \sigma(\mathrm{SM})$, where $\sigma(\mathrm{SM})$ is the total SM Higgs boson production cross section, are determined at the $95 \%$ CL. Right: Limits on the spin-independent DM-nucleon scattering cross section in Higgs-portal models assuming a scalar (blue) or fermion (red) DM particle. The limits are given at the $90 \%$ CL to allow for comparison to direct detection constraints from the LUX (green), PandaX- II (purple), and CDMSlite (light blue) experiments.

\section{Search for new light bosons in decays of the $h(125)$}

\section{1 $\mathbf{h} \rightarrow a a \rightarrow 4 \mu$}

A search for BSM decays of the $\mathrm{h}(125)$ to pairs of new light bosons, which subsequently decay to pairs of oppositely charged muons ( $h \rightarrow 2 a+X \rightarrow 4 \mu+X)$ has been performed. This search is based on a data sample corresponding to an integrated luminosity of $2.8 \mathrm{fb}^{-1}$ at $\sqrt{s}=$ 
$13 \mathrm{TeV}$ in 2015. One event with two dimuons of consistent single mass in the signal region was observed. The analysis has been designed as a model-independent search allowing interpretation of its results in the context of a broad range of new physics scenarios predicting the same type of final state signature. The results are also interpreted in the context of the NMSSM and the dark SUSY benchmark models for $m_{\mathrm{h}}<150 \mathrm{GeV}$, as shown in Fig. 3 [13]. This search constrains a large, previously unconstrained area of the parameter space.
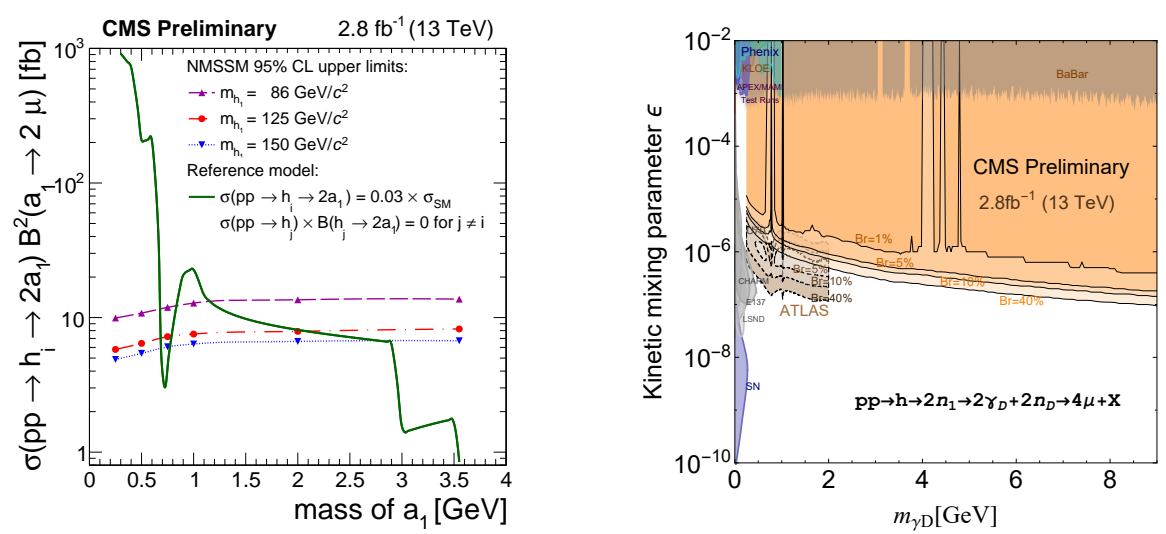

Figure 3: Left: The 95\% CL upper limits as functions of $m_{a_{1}}$, for the NMSSM case, on $\sigma\left(p p \rightarrow h_{1,2} \rightarrow 2 a_{1}\right)$ x $B^{2}\left(a_{1} \rightarrow 2 \mu\right)$ with $m_{h_{1}}=86 \mathrm{GeV}$ (dashed curve), $m_{h_{1}}=125 \mathrm{GeV}$ (dash dotted curve), and $m_{h_{1}}=150 \mathrm{GeV}$ (dotted curve). Right: 95\% CL upper limits (black solid curves) from this search on $\sigma(p p \rightarrow \mathrm{h}) \cdot \mathrm{B}(\mathrm{h}$ $\left.\rightarrow 2 \gamma_{D}+X\right)$ (with $m_{n_{1}}=10 \mathrm{GeV}, m_{n_{D}}=1 \mathrm{GeV}$ ) in the plane of two parameters $\left(\varepsilon\right.$ and $\left.m_{\gamma_{D}}\right)$ for the dark SUSY scenarios, along with constraints from other experiments.

\subsection{Other $\mathbf{h} \rightarrow a a$ searches}

Further searches for the decay of the SM-like Higgs boson to pairs of light scalars were performed using $19.7 \mathrm{fb}^{-1}$ of pp collisions at a centre-of-mass energy of $8 \mathrm{TeV}$ in final states with taus, muons or $b$ quark jets. The data were found to be compatible with SM predictions. Direct limits are set on the cross section times branching fraction for several signal processes. According to the 2HDM and extensions, the ratio of the decay widths of a light scalar boson to different types of leptons only depends on the masses of these leptons. A similar relation also exists between the partial decay widths of the light scalar boson to leptons and down-type quarks, only in Type-1 and Type-2. Therefore, the results of the SM-like Higgs boson to pairs of light bosons in different channels are interpreted and compared in different scenarios of two-Higgs-doublet models with an additional scalar singlet (2HDM+S) shown in Fig. 4.

\section{Search for Lepton Flavour Violation of the $h(125)$}

A direct search for lepton flavour violating decays of the Higgs boson in the $\mathrm{h} \rightarrow \mu \tau, e \tau$ and $e \mu$ channels is performed using data collected by the CMS experiment with corresponding integrated luminosity of $2.3 \mathrm{fb}^{-1}$ at $\sqrt{s}=13 \mathrm{TeV}$ in the case of $\mathrm{h} \rightarrow \mu \tau$ and $19.7 \mathrm{fb}^{-1}$ at $\sqrt{s}=8 \mathrm{TeV}$ in the case of $\mathrm{h} \rightarrow e \tau / e \mu$. The measurements are sensitive to the branching fraction of lepton flavour 


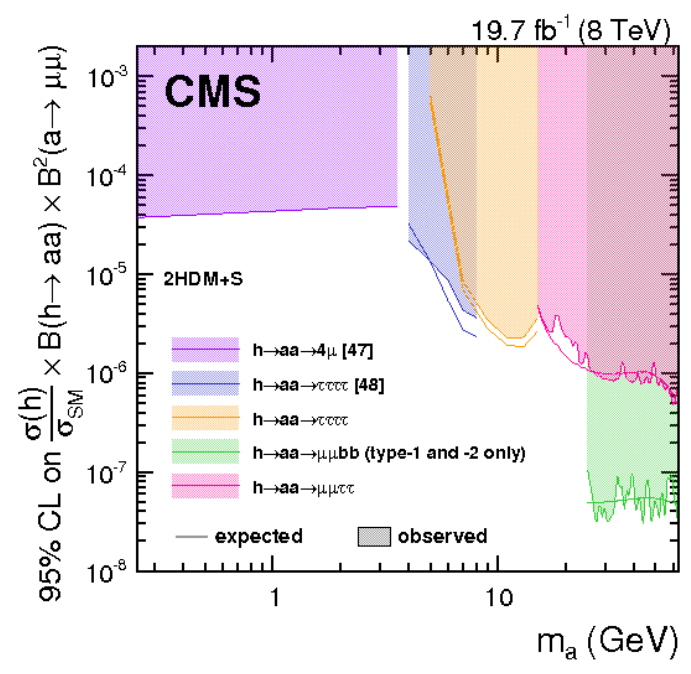

Figure 4: Expected and observed 95\% CL exclusion limits on $\left(\sigma_{h} / \sigma_{S M}\right) \times \mathrm{B}(\mathrm{h} \rightarrow a a) \times \mathrm{B}^{2}(a \rightarrow \mu \mu)$ for various exotic $\mathrm{h}(125)$ boson decay searches, assuming that the branching fractions of the light scalar boson to muons, $\tau$ leptons and $\mathrm{b}$ quarks. This assumption implies that the limit shown for $\mathrm{h} \rightarrow a a \rightarrow \mu \mu \mathrm{bb}$ is valid only in type-1 and type-2 $2 \mathrm{HDM}+\mathrm{S}$ models.

violating decays of the Higgs boson to each channel. No excess is observed. Upper limits at $95 \%$ confidence level on the branching fractions $\mathrm{B}(\mathrm{h} \rightarrow \mu \tau)<1.20 \%$ (1.62\% expected), $\mathrm{B}(\mathrm{h} \rightarrow e \tau)<$ $0.69 \%$ ( $0.75 \%$ expected) and $\mathrm{B}(\mathrm{h} \rightarrow e \mu)<0.035 \%$ ( $0.048 \%$ expected) are obtained. Moreover, the constraints on branching fraction can be interpreted in terms of LFV Yukawa couplings. The LFV decays $\mathrm{h} \rightarrow e \mu, e \tau, \mu \tau$ would arise at tree level from the assumed flavour violating Yukawa interactions, $Y_{\ell^{\alpha} \ell^{\beta}}$ where $\ell^{\alpha}, \ell^{\beta}$ denote the leptons, $\ell^{\alpha}, \ell^{\beta}=e, \mu, \tau$ and $\ell^{\alpha} \neq \ell^{\beta}$. The 95\% CL upper limits on the Yukawa couplings derived for each branching fraction are shown in Fig. 5 [14, 15].
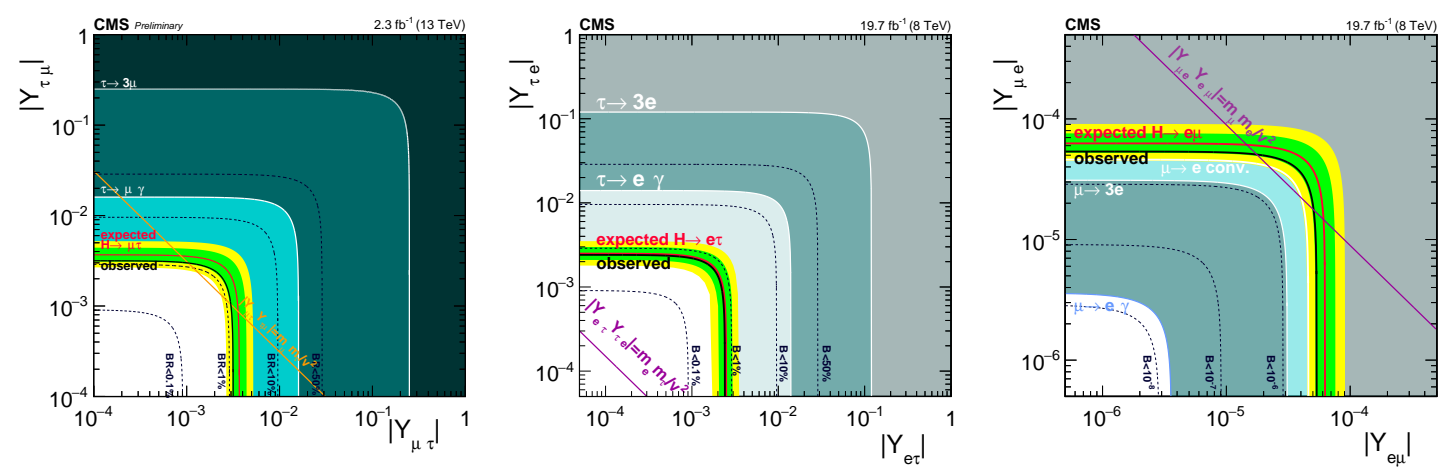

Figure 5: Constraints on the flavour violating Yukawa couplings $\left|Y_{\mu \tau}\right|,\left|Y_{\tau \mu}\right|$ (left), $\left|Y_{e \tau}\right|,\left|Y_{\tau e}\right|$ (middle), $\left|Y_{e \mu}\right|,\left|Y_{\mu e}\right|$ (right). The expected (red solid line) and observed (black solid line) limits are derived from the limits on the branching fractions. The green (yellow) band indicates the range that is expected to contain $68 \%(95 \%)$ of the statistical excursions of the limit. 


\section{Summary}

Several searches for BSM phenomena in decays of the discovered Higgs boson at $125 \mathrm{GeV}$ have been performed in CMS experiment since Run-I and are being continued in 2015 data. No evidence of new BSM particle production has been observed so far. The upper limits are set on the branching fraction of the Higgs boson decay to different BSM particles, as well as lepton flavour violating decays of the Higgs boson. The results are interpreted in terms of several BSM theories, including Higgs-portal model of DM interactions, 2HDM and its extension especially NMSSM.

All the presented searches are being continued with the full 2016 Run-II data, in which the integrated luminosity has already reached $36 \mathrm{fb}^{-1}$. This amount of integrated luminosity will give a further substantial boost to the sensitivity for new physics phenomena in Higgs boson decays.

\section{References}

[1] ATLAS Collaboration, Observation of a new particle in the search for the Standard Model Higgs boson with the ATLAS detector at the LHC, Phys. Lett. B 716 (2012) 1.

[2] CMS Collaboration, Observation of a new boson at a mass of $125 \mathrm{GeV}$ with the CMS experiment at the LHC, Phys. Lett. B 716 (2012) 30.

[3] CMS Collaboration, Observation of a new boson with mass near $125 \mathrm{GeV}$ in pp collisions at $\sqrt{s}=7$ and $8 \mathrm{TeV}, \mathrm{JHEP} 06$ (2013) 081.

[4] ATLAS and CMS Collaboration, Measurements of the Higgs boson production and decay rates and constraints on its couplings from a combined ATLAS and CMS analysis of the LHC pp collision data at $\sqrt{s}=7$ and $8 \mathrm{TeV}, \mathrm{JHEP} \mathbf{0 8}$ (2016) 045.

[5] D. Curtin, R. Essig, and Y.-M. Zhong, Uncovering light scalars with exotic Higgs decays to bb $\mu \mu$, JHEP 06 (2015) 025.

[6] B. McWilliams and L.-F. Li, Virtual effects of Higgs particles, Nucl. Phys. B 179 (1981) 62.

[7] O. U. Shanker, Flavor violation, scalar particles and leptoquarks, Nucl. Phys. B 206 (1982) 253.

[8] CMS Collaboration, VBF Higgs invisible search with 2015 data, CMS-PAS-HIG-16-009.

[9] CMS Collaboration, Search for dark matter in Z(ll)+MET final state using the 2016 dataset, CMS-PAS-EXO-16-038.

[10] CMS Collaboration, Search for dark matter in final states with an energetic jet, or a hadronically decaying $W$ or $Z$ boson using $12.9 \mathrm{fb}^{-1}$ of data at $\sqrt{\mathrm{s}}=13 \mathrm{TeV}$, CMS-PAS-EXO-16-037, arXiv:1703.01651.

[11] A. Djouadi, O. Lebedev, Y. Mambrini, and J. Quevillon, Implications of LHC searches for Higgs-portal dark matter, Phys. Lett. B 709 (2012) 65.

[12] CMS Collaboration, A combination of searches for invisible Higgs boson decays using run I and run II data samples, JHEP 02 (2017) 135.

[13] CMS Collaboration, A search for beyond the standard model light bosons decaying into muon pairs with $13 \mathrm{TeV}$ data, CMS-PAS-HIG-16-035.

[14] CMS Collaboration, Search for lepton flavour violating decays of the Higgs boson to e $\tau$ and e $\mu$ in proton-proton collisions at $\sqrt{s}=8$ TeV, Phys. Lett. B 763 (2016) 472.

[15] CMS Collaboration, Search for lepton flavour violating decays of the Higgs boson in the $\mu \tau$ final state at $13 \mathrm{TeV}$, CMS-PAS-HIG-16-005. 